\title{
Erythrocyte deformability, plasma lipid peroxidation and plasma protein oxidation in a group of OSAS subjects
}

\author{
G. Caimi*, M. Montana, B. Canino, V. Calandrino, R. Lo Presti and E. Hopps \\ Dipartimento Biomedico di Medicina Interna e Specialistica, Universitá di Palermo, Italy
}

\begin{abstract}
Considering that obstructive sleep apnea syndrome (OSAS) is usually associated with endothelial dysfunction, atherosclerosis and cardiovascular disorders, our aim was to examine the erythrocyte deformability and the oxidative status in a group of OSAS subjects. We consecutively enrolled 48 subjects with OSAS defined after a 1-night cardiorespiratory sleep study, subsequently subdivided according to the apnea/hypopnea index (AHI) value in two subgroups: Low (L=21 subjects with $\mathrm{AHI}<30)$ and High $(\mathrm{H}=27$ subjects with $\mathrm{AHI}>30)$. We evaluated the erythrocyte deformability, expressed as elongation index (EI) and the parameters of the oxidative status, such as lipid peroxidation (expressed as thiobarbituric acid-reactive substances - TBARS) and protein oxidation (measured as carbonyl groups - PC). In the entire group and in the two subgroups of OSAS subjects we found a decreased erytrocyte deformability at all shear stresses, not correlated with the plasmatic oxidative stress nor with the polysomnographic parameters. Lipid peroxidation was increased in the whole group and in the H subgroup of OSAS while protein oxidation showed a different trend. As in OSAS the osmotic fragility and the metabolism of the red cells seem to be not impaired, the oxidative damage to the red cell membrane proteins might be responsible for the reduced erythrocyte deformability. This rheological alteration, in addition to the increase in whole blood and plasma viscosity and to the erythrocyte hyperaggregation, could influence the microcircolatory profile in OSAS subjects.
\end{abstract}

Keywords: Erythrocyte deformability, lipid peroxidation, protein oxidation, OSAS

\section{Introduction}

Obstructive sleep apnea syndrome (OSAS) is characterized by repeated partial or complete obstructions of upper airways, usually occurring at the level of the oropharynx, during sleep with resulting apnea or hypopnea, with consequent intermittent arterial oxygen desaturation and sleep disruption [11]. The intermittent hypoxemia can increase sympatetic tone, oxidative stress, pro-inflammatory cytokine production, platelet aggregation, and metabolic dysregulation [15]. OSAS is significantly and independently associated with an increased risk of cardiovascular diseases, cerebrovascular events and all-cause mortality and the incidence of cardiovascular events seems to be related to OSAS severity [32]. Subjects with severe untreated OSAS have an higher incidence of fatal and non fatal cardiovascular events in comparison with simple snorers or OSAS patients treated with cPAP [18]. Yaggi et al. [33] demonstrated that OSAS subjects had a 2-fold increased risk of stroke and this association was independent from other cardiovascular and cerebrovascular risk factors, such as hypertension. Some papers have evaluated the effects of OSAS on blood rheology. Chin et al. [3] showed increased levels of fibrinogen and hematocrit in the morning in a small group of OSAS subjects, suggesting an increment

Corresponding author: Gregorio Caimi, Dipartimento Biomedico di Medicina Interna e Specialistica, Universitá degli Studi di Palermo, Via del vespro 129, 90100 Palermo, Italy. Tel.: +39 091 6554406; Fax: +39 091 6554535; E-mail: gregorio.caimi@unipa.it. 
in blood viscosity. Also Nobili et al showed an increased plasma fibrinogen, which was correlated with AHI value and with nocturnal minimal oxygen saturation $\left(\mathrm{SO}_{2}\right)$, and an increased blood viscosity [20]. Tazbirek et al found elevated blood viscosity and erythrocyte aggregation in obese men with OSAS in comparison with those without OSAS [29]. However, other authors [9] observed an increase only in plasma viscosity, inversely correlated with mean nocturnal $\mathrm{SO}_{2}$, but no modification of erythrocyte deformability (evaluated with filtration techniques). Peled et al demonstrated that in OSAS subjects the increased erythrocyte aggregation is correlated with PCR levels [22], while Sinnapah et al. [25] found a correlation between erythrocyte aggregation and AHI and between erythrocyte aggregation and BMI in overweight OSAS subjects. This last paper suggested that BMI is more predictive of erythrocyte aggregation than AHI and that overweight influences blood rheology more than OSAS severity [25].

The treatment with continuous positive airways pressure (cPAP) during the sleep reduces plasma fibrinogen [3], and blood and plasma viscosity [29] improving the blood rheological properties. Reinhart et al. observed a significant reduction of plasma viscosity and platelet activation after six months of cPAP therapy [23], while other authors demonstrated an improvement in hematocrit values, blood viscosity and platelet aggregation after only 30 days of treatment [34].

Keeping in mind all these considerations, our aim was the evaluation of the erythrocyte deformability, expressed as elongation index (EI), and especially the analysis of the influence of the polysomnographic parameters, of the lipid peroxidation and of the protein oxidation on this determinant of the hemorheological profile in our group of OSAS subjects.

\section{Subjects}

We examined 48 subjects (36 men and 12 women; mean age $50.3 \pm 14.68$ yrs) with obstructive sleep apnea from those with suspected OSAS referred to our center. Clinical history and physical examination were performed in all subjects and Epworth Sleepiness Scale (ESS) was also given. OSAS was diagnosed after a 1-night cardiorespiratory sleep study and its severity was assessed evaluating the apnea/hypopnea index (AHI); we also defined the oxygen desaturation index (ODI) and the nocturnal mean oxygen saturation $\left(\mathrm{mSO}_{2}\right)$. OSAS subjects were subdivided according to the AHI value in two subgroups: Low $(L=21$ subjects with AHI $<30)$ and High $(H=27$ subjects with AHI $>30)$, therefore the Low subgroup included subjects with mild to moderate OSAS, while the H subgroup included the subjects with severe OSAS. The mean age in the L subgroup was $45.3 \pm 14.4$, while in the H subgroup was $52.8 \pm 14.2$ yrs. The mean ESS score in the entire group of OSAS subjects was $11.1 \pm 5.1$, in the $\mathrm{L}$ subgroup was $9.2 \pm 3.7$, while in the $\mathrm{H}$ subgroup was $12.4 \pm 5.6$. Means and S.D. of BMI, waist circumference, neck circumference, and polysomnographic parameters are reported in Table 1 (Table 1). All the subjects gave their informed consent before entering the study and the study was approved by the Ethical Committee.

\section{Methods}

Venous blood samples were collected in the morning by venous puncture from the antecubital vein of fasting subjects and immediately transferred to anticoagulated glass tubes for evaluation of erythrocyte deformability, lipid peroxidation and protein oxidation.

\subsection{Elongation index (EI)}

To evaluate erythrocyte deformability, we mixed $30 \mu \mathrm{l}$ of anticoagulated blood with $2 \mathrm{ml}$ of dextran solution at a viscosity of $24 \mathrm{mPa}$. The measurement was obtained by using the diffractometer Rheodyn 
Table 1

Mean \pm S.D. of age, anthropometric characteristics and OSAS parameters in the entire group of OSAS patients and in the two subgroups with respectively mild and severe disease

\begin{tabular}{lccc}
\hline & All OSAS patients & L subgroup & H subgroup \\
\hline Anthropometric characteristics & & & \\
BMI $\left(\mathrm{kg} / \mathrm{m}^{2}\right)$ & $35.4 \pm 7.3$ & $35.7 \pm 8.5$ & $35.1 \pm 6.5$ \\
Waist circumference (cm) & $118.8 \pm 16.1$ & $114.2 \pm 14.5$ & $122.5 \pm 16.6$ \\
Neck circumference (cm) & $44.4 \pm 4.5$ & $41.5 \pm 3.2$ & $46.6 \pm 4.1$ \\
Polysomnographyc parameters & & & \\
AHI & $38.5 \pm 25.7$ & $15.1 \pm 8.1$ & $56.6 \pm 18.9$ \\
$\mathrm{mSO}_{2}(\%)$ & $91.1 \pm 3.7$ & $93.4 \pm 2.7$ & $89.5 \pm 3.4$ \\
$\mathrm{ODI}$ & $39.3 \pm 29.0$ & $14.3 \pm 9.4$ & $55.4 \pm 25.7$ \\
\hline
\end{tabular}

${ }^{\S} p<0.001$ vs L subgroup (Student's $t$ test for unpaired data). BMI = Body Mass Index. $\mathrm{mSO}_{2}=$ mean oxygen saturation. $\mathrm{AHI}=$ apnea/hypopnea index. $\mathrm{ODI}=$ oxygen desaturation index.

SSD of Myrenne, which measures the diffraction pattern of a laser beam passing through erythrocytes suspended in a viscous medium and deformed by a force with defined shear stress. The shear stresses employed by us were $6,12,30$ and $60 \mathrm{~Pa}$. The erythrocyte deformation was expressed as elongation index $(E I)=(l-w / l+w) \times 100$, were $l=$ length and $w=$ width of the erythrocytes.

\subsection{Lipid peroxidation}

Lipid peroxidation was evaluated in plasma by detection of thiobarbituric acid-reactive substances (TBARS), generated by peroxidative processes, which include lipid peroxides and malonildialdehyde. The evaluation of TBARS was made by fluorimetry, using 1,1,3,3-tetramethoxypropane as standard.

\subsection{Protein oxidation}

Protein oxidation was evaluated as protein carbonyl groups (PC). The PC groups were measured by an enzyme-linked immunosorbent assay (ELISA) kit (BioCell PC test kit, Enzo Life Sciences AG, Switzerland).

Regarding the erythrocyte deformability the control group included 45 subjects (20 women and 25 men, mean age $37.7 \pm 7.8$ years), while regarding the lipid and protein oxidation the control group included 59 subjects (16 women and 43 men, mean age $36.2 \pm 8.2$ years); both the groups of controls were free of medical diseases as assessed by clinical history, physical examination, electrocardiography, and routine hematological and urine analysis.

\subsection{Statistical analysis}

Data were expressed as means \pm S.D.; the difference between normal controls and OSAS subjects was evaluated according to the Student's $t$ test for unpaired data. The statistical difference between normal subjects and OSAS subjects subdivided according to the apnea/hypopnea index (AHI) was estimated using the 1-way analysis of variance (ANOVA) integrated with the Bonferroni test. The correlations were performed employing the linear regression test. The null hypothesis was rejected for $p$ values $<0.05$. 
Table 2

Means \pm S.D. of the rheological and oxidative parameters in control subjects and in the entire group of OSAS patients

\begin{tabular}{lcc}
\hline & Control subjects & All OSAS patients \\
\hline EI 6 Pa & $27.3 \pm 5.7$ & $21.8 \pm 4.8^{\S}$ \\
EI $12 \mathrm{~Pa}$ & $35.2 \pm 5.5$ & $29.9 \pm 4.7^{\S}$ \\
EI $30 \mathrm{~Pa}$ & $43.0 \pm 5.0$ & $39.2 \pm 4.3^{\S}$ \\
EI $60 \mathrm{~Pa}$ & $46.2 \pm 4.5$ & $43.2 \pm 3.6^{\#}$ \\
TBARS (nmol/ml) & $5.71 \pm 1.42$ & $6.43 \pm 1.63^{*}$ \\
PC (nmol/mg prot.) & $0.347 \pm 0.094$ & $0.316 \pm 0.120$ \\
\hline
\end{tabular}

${ }^{*} p<0.05$. ${ }^{*} p<0.01 .^{\S} p<0.001$ vs control subjects (Student's $t$ test). OSAS $=$ Obstructive Sleep Apnea Syndrome. $\mathrm{EI}=$ Elongation Index. TBARS $=$ ThioBarbituric Acid Reactive Substances. PC $=$ Protein Carbonyls.

Table 3

Means \pm S.D. of the rheological and oxidative parameters in control subjects and in the two subgroups of OSAS patients with respectively mild and severe disease

\begin{tabular}{lcccc}
\hline & Control subjects & L subgroup & H subgroup & F \\
\hline EI 6 Pa & $27.3 \pm 5.7$ & $21.8 \pm 5.8^{\#}$ & $21.8 \pm 4.1^{\S}$ & $10.32^{\mathrm{a}}$ \\
EI 12 Pa & $35.2 \pm 5.5$ & $29.9 \pm 5.9^{\#}$ & $29.9 \pm 3.8^{\S}$ & $10.30^{\mathrm{a}}$ \\
EI 30 Pa & $43.0 \pm 5.0$ & $38.4 \pm 5.2^{\#}$ & $39.6 \pm 3.7^{*}$ & $7.00^{\mathrm{b}}$ \\
EI 60 Pa & $46.2 \pm 4.5$ & $42.5 \pm 4.4^{*}$ & $43.5 \pm 3.0^{*}$ & $5.86^{\mathrm{b}}$ \\
TBARS (nmol/ml) & $5.71 \pm 1.42$ & $5.25 \pm 0.47$ & $7.35 \pm 1.63^{\text {§ศ }}$ & $18.0^{\mathrm{a}}$ \\
PC (nmol/mg prot.) & $0.347 \pm 0.094$ & $0.230 \pm 0.088$ & $0.382 \pm 0.099^{\natural}$ & $16.6^{\mathrm{a}}$ \\
\hline
\end{tabular}

${ }^{\mathrm{a}} p<0.001$. ${ }^{\mathrm{b}} p<0.01$. (ANOVA). ${ }^{*} p<0.05$. ${ }^{*} p<0.01 .{ }^{\S} p<0.001$ vs control subjects (Bonferroni's test). " $p<0.001 \mathrm{vs} \mathrm{L}$ subgroup (Bonferroni's test). OSAS = Obstructive Sleep Apnea Syndrome. AHI = Apnea-Hypopnea Index. EI = Elongation Index. TBARS $=$ ThioBarbituric Acid Reactive Substances. $\mathrm{PC}=$ Protein Carbonyls.

\section{Results}

In the whole group of OSAS subjects we found a decrease in the elongation index at all the shear stresses in comparison with normal controls (Table 2). In the same group of OSAS we found a significant increase in lipid peroxidation, while no difference was noted about protein oxidation (Table 2). Subdividing the entire group of OSAS according to the AHI value we found in the two subgroups a significant decrease in EI in comparison with normal controls (Table 3), while regarding the lipid peroxidation we found its increase only in the H subgroup of OSAS subjects and about the protein oxidation we noted a decrease in the L subgroup and an increase in the $\mathrm{H}$ subgroup compared to normal controls (Table 3).

In the whole group of OSAS subjects no correlation among EI, at all the shear stresses, AHI, mean nocturnal $\mathrm{SO}_{2}$ and ODI was observed, and the same trend was present in the two subgroups. In the entire group of OSAS subjects no correlation among EI and lipid peroxidation was noted (Fig. 1) and the same behavior was evident in the two subgroups. No correlation among elongation index, at all the shear stresses, and protein oxidation was found in the whole group of OSAS subjects (Fig. 1) nor in the two subgroups. 

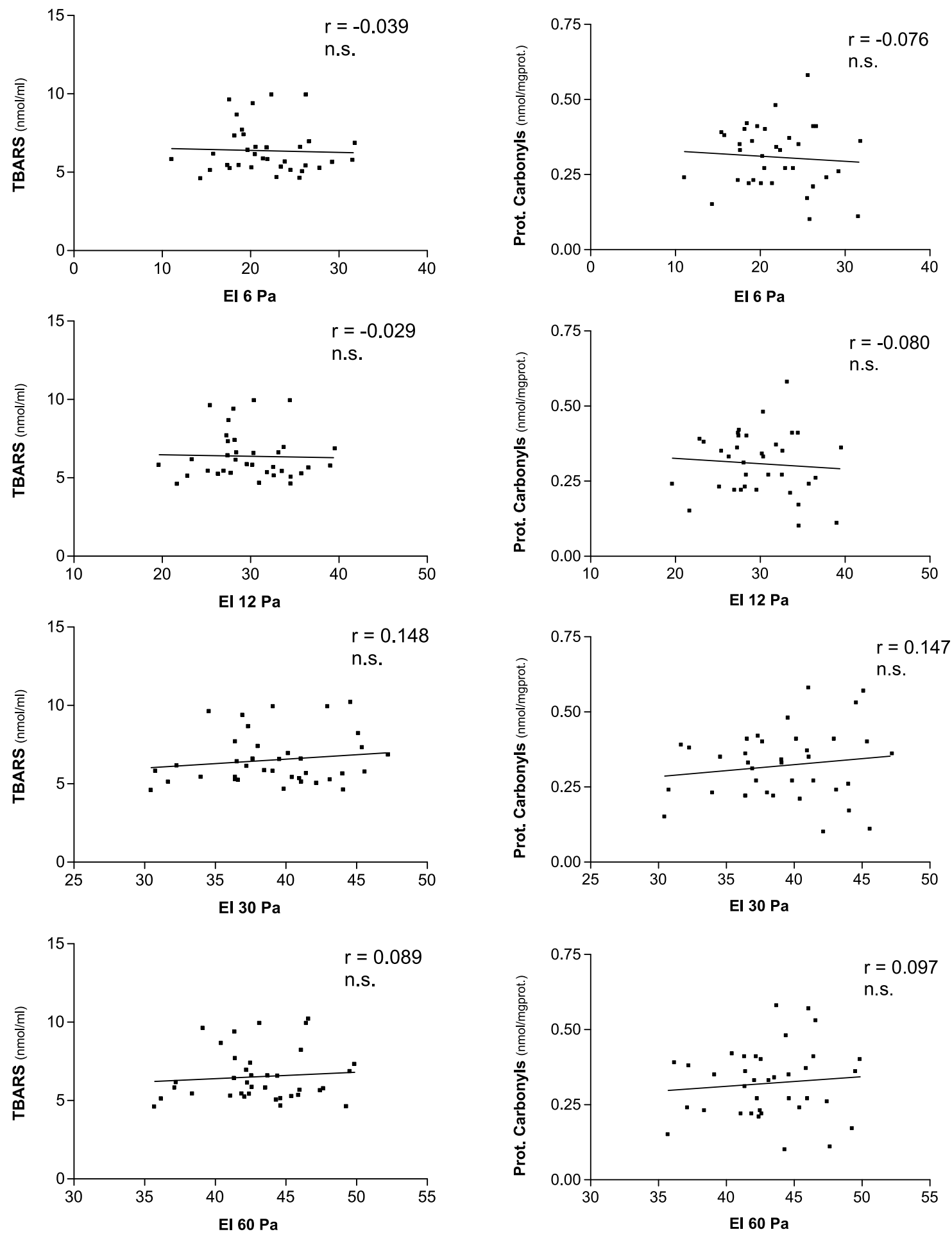

Fig. 1. Correlations between Elongation Index and parameters of oxidative stress in the entire group of OSAS patients. 


\section{Discussion}

Previously in the same group of OSAS subjects we examined the behavior of the nitric oxide metabolites and of the erythrocyte deformability [2] as well as the behavior of lipid peroxidation and protein oxidation [13]. Now therefore our purpose has been to look for some possible mechanisms able to explain the reduction of erythrocyte deformability in this clinical condition. Our datum concerning the erythrocyte deformability in OSAS is different from which of other authors $[9,25,29]$. In this brief report no statistical correlation among erythrocyte deformability and polysomnographic parameters was observed although others [29] have described a negative correlation between this rheological parameter and the nocturnal minimal $\mathrm{SO}_{2}$.

In this clinical condition the investigations regarding the osmotic fragility of red cells [21], the erythrocyte metabolism $[9,17,21]$, and the erythrocyte lipid peroxidation [9, 21] have resulted negative and this finding has directed our attention to the evaluation of extra-erythrocyte factors, such as the parameters reflecting plasma lipid peroxidation and protein oxidation. In our experience these two parameters result significantly correlated with the polysomnographic profile in OSAS subjects [13]. However, in the same group of OSAS subjects no correlation between erythrocyte deformability and plasma lipid peroxidation nor between erythrocyte deformability and plasma protein oxidation has been demonstrated.

As it is known, the erythrocyte deformability depends on the surface to volume ratio, the internal viscosity and the membrane dynamic properties [24]. Considering that the internal viscosity is related especially to the erythrocyte metabolism and that this metabolic aspect results to be normal in OSAS $[9,17,21]$, we must retain that this alteration could be ascribed to the abnormality of the membrane dynamic properties. These properties depend expecially on the cholesterol/phospholipid ratio, on the saturated/unsaturated fatty acid ratio, and on the membrane proteins.

The data up to now published underline that in OSAS an alteration of the lipid profile of the erythrocyte membrane, expressed as lipid peroxidation, is not present $[9,21]$ and then it is possible that the impairment of the erythrocyte deformability might be due to an alteration of the membran proteins. This first supposition is based on the consideration that OSAS, characterized by short and repetitive cycles of hypoxia and reoxygenation, activates significantly several inflammatory mechanisms $[1,12]$ such as CRP, TNF- $\alpha$, IL-8, and IL-6 [7, 10, 14, 19] that may modify the erythrocyte membrane dynamic properties. Among these biomarkers increased in OSAS we believe that a possible role may be carried out by IL-8. Up to now in fact there are some data regarding its significant increase in the membrane of circulating red cells in acute clinical conditions $[8,30]$ and then an IL-8 increase could be plausible also in erythrocyte membranes of OSAS subjects. Another hypothesis may regard the possible damage caused by the oxidative stress on these dynamic membrane properties. Some ROS, such as hydrogen peroxide [26] and superoxide anion [31], seem to influence the erythrocyte deformability damaging the membrane proteins. As regards, we must underline that Davies et al. [5, 6] have assured that the oxidative damage of the membrane proteins is a more sensitive indicator of oxidative stress than lipid peroxidation. The last supposition that could explain the trend of erythrocyte deformability in OSAS might be identified in the variation of $\mathrm{pH}$ that seems to accompany this syndrome. During the cycles of hypoxia and reoxygenation a condition of respiratory acidosis may also be present and may influence the red cell rheology; there are many findings regarding the effect of the $\mathrm{pH}$ on elastic deformability of the erythrocyte membrane $[4,16]$, although in our group of OSAS subjects we have no information about the acid-base balance.

Although it is not easy to ascertain the causes of the decrease of erythrocyte deformability in OSAS, we must take account of this rheological datum that together with other rheological alterations observed in this clinical condition, such as the increase in whole blood viscosity [20], especially at morning, 
in plasma viscosity and erythrocyte aggregation [9, 22, 25, 29], delineate a picture of hyperviscosity syndrome of which must allow considering the prognosis of these subjects.

This research complies with the requirement for ethical publication in Clinical Hemorheology and Microcirculation as published in Clin Hemorheol Microcirc. 2010;44(1):1-2.

\section{References}

[1] K. Archontogeorgis, E. Nena, N. Papanas and P. Steiropoulos, Biomarkers to improve diagnosis and monitoring of obstructive sleep apnea syndrome: Current status and future perspectives, Pulmonary Medicine 2014 (2014), 930535.

[2] B. Canino, E. Hopps, V. Calandrino, M. Montana, R. Lo Presti and G. Caimi, Nitric oxide metabolites and erythrocyte deformability in a group of subjects with obstructive sleep apnea syndrome, Clinical Hemorheology and Microcirculation 59 (2015), 45-52.

[3] K. Chin, M. Ohi, H. Kita, T. Noguchi, N. Otsuka, T. Tsuboi, M. Mishima and K. Kuno, Effects of NCPAP therapy on fibrinogen levels in obstructive sleep apnea syndrome, American Journal of Respiratory Critical Care Medicine 153 (1996), 1972-1976.

[4] E.D. Crandall, A.M. Critz, A.S. Osher, D.J. Keljo and R.E. Forster, Influence of pH on elastic deformability of the human erythrocyte membrane, American Journal of Physiology 235 (1978), C269-C278.

[5] K.J. Davies and A.L. Goldberg, Oxygen radicals stimulate intracellular proteolysis and lipid peroxidation by independent mechanisms in erythrocytes, The Journal of Biological Chemistry 262 (1987), 8220-8226.

[6] K.J. Davies and A.L. Goldberg, Proteins damaged by oxygen radicals are rapidly degraded in extracts of red blood cells, The Journal of Biological Chemistry 262 (1987), 8227-8234.

[7] M. de la Peña, A. Barceló, F. Barbe, J. Piérola, J. Pons, E. Rimbau, O. Ayllón and A.G. Agustí, Endothelial function and circulating endothelial progenitor cells in patients with sleep apnea syndrome, Respiration 76 (2008), 28-32.

[8] R.J. de Winter, A. Manten, Y.P. de Jong, R. Adams, S.J. van Deventer and K.I. Lie, Interleukin 8 released after acute myocardial infarction is mainly bound to erythrocytes, Heart 78 (1997), 598-602.

[9] N. Dikmenoglu, B. Ciftci, E. Ileri, S.F. Guven, N. Seringec, Y. Aksoy and D. Ercil, Erythrocyte deformability, plasma viscosity and oxidative status in patients with severe obstructive sleep apnea syndrome, Sleep Medicine 7 (2006), 255-261.

[10] A.A. El Solh, M.E. Akinnusi, F.H. Baddoura and C.R. Mankowski, Endothelial cell apoptosis in obstructive sleep apnea: A link to endothelial dysfunction, American Journal for Respiratory and Critical Care Medicine 175 (2007), 1186-1191.

[11] L.J. Epstein, D. Kristo, P.J. Strollo Jr, N. Friedman, A. Malhotra, S.P. Patil, K. Ramar, R. Rogers, R.J. Schwab, E.M. Weaver and M.D. Weinstein, Adult Obstructive Sleep Apnea Task force of the American Academy of Sleep Medicine, Clinical guidelines for the evaluation, management and long-term care of obstructive sleep apnea syndrome, Journal of Clinical Sleep Medicine 5 (2009), 263-276.

[12] J.F. Garvey, C.T. Taylor and W.T. McNicholas, Cardiovascular disease in obstructive sleep apnoea syndrome: The role of intermittent hypoxia and inflammation, European Respiratory Journal 33 (2009), 1195-1205.

[13] E. Hopps, B. Canino, V. Calandrino, M. Montana, R. Lo Presti and G. Caimi, Lipid peroxidation and protein oxidation are related to the severity of OSAS, European Review for Medical and Pharmacological Sciences 18 (2014), 3773-3778.

[14] C.M. Hoyos, K.L. Melehan, P.Y. Liu, R.R. Grunstein and C.L. Phillips, Does obstructive sleep apnea cause endothelial dysfunction? A critical review of the literature, Sleep Medicine Reviews 20C (2015), 15-26.

[15] B.D. Kent, S. Ryan and W.T. McNicholas, Obstructive sleep apnea and inflammation: Relationship to cardiovascular co-morbidity. Respiratory Physiology \& Neurobiology 178 (2011), 475- 481.

[16] D. Kuzman, T. Znidarcic, M. Gros, S. Vrhovec, S. Svetina and B. Zeks, Effect of pH on red blood cell deformability, Pflugers Archive 440 (2000), R193-R194.

[17] D. Maillard, B. Fleury, B. Housset, S. Laffont, J. Chabolle and J.P.H. Derenne, Decreased oxyhemoglobin affinity in patients with sleep apnea syndrome, American Review for Respiratory Diseases 143 (1991), 486-489.

[18] J.M. Marin, S.J. Carrizo, E. Vicente and A.G. Agusti, Long-term cardiovascular outcomes in men with obstructive sleep apnoea-hypopnoea with or without treatment with continuous positive airway pressure: An observational study, Lancet 365 (2005), 1046-1053.

[19] K. Martin, M. Stanchina, N. Kouttab, E.O. Harrington and S. Rounds, Circulating endothelial cells and endothelial progenitor cells in obstructive sleep apnea, Lung 186 (2008), 145-150.

[20] L. Nobili, G. Schiavi, E. Bozano, F. De Carli, F. Ferrillo and F. Nobili, Morning increase of whole blood viscosity in obstructive sleep apnea syndrome, Clinical Hemorheology and Microcirculation 22 (2000), 21-27. 
[21] L. Ozturk, B. Mansour, M. Yuksel, A.S. Yalcin, F. Celikoglu and N. Gokhan, Lipid peroxidation and osmotic fragility of red blood cells in sleep-apnea patients, Clinica Chimica Acta 332 (2003), 83-88.

[22] N. Peled, M. Kassirer, M.R. Kramer, O. Rogowski, D. Shlomi, B. Fox, A.S. Berliner and D. Shitrit, Increased erythrocyte adhesiveness and aggregation in obstructive sleep apnea syndrome, Thrombosis Research 121 (2008), 631-636.

[23] W.H. Reinhart, J. Oswald, R. Walter and M. Kuhn, Blood viscosity and platelet function in patients with obstructive sleep apnea syndrome treated with nasal continuous positive airway pressure, Clinical Hemorheology and Microcirculation 27 (2002), 201-207.

[24] T. Shiga, N. Maeda and K. Kon, Erythrocyte rheology, Critical Reviews in Oncology/Hematology 10 (1990), 9-48.

[25] S. Sinnapah, G. Cadelis, Y. Lamarre and P. Connes, Overweight explains the increased red blood cell aggregation in patients with obstructive sleep apnea, Clinical Hemorheology and Microcirculation 59 (2015), 17-26.

[26] L.M. Snyder, N.L. Fortier, J. Trainor, J. Jacobs, L. Leb, B. Lubin, D. Chiu, S. Shohet and N. Mohandas, Effect of hydrogen peroxide exposure on normal human erythrocyte deformability, morphology, surface characteristics, and spectrin-hemoglobin cross-linking, Journal of Clinical Investigation 76 (1985), 1971-1977.

[27] S. Steiner, T. Jax, S. Evers, M. Hennersdorf, A. Schwalen and B.E. Strauer, Altered blood rheology in obstructive sleep apnea as a mediator of cardiovascular risk, Cardiology 104 (2005), 92-96.

[28] M. Tazbirek, L. Slowinska, M. Kawalski and W. Pierzchala, The rheological properties of blood and the risk of cardiovascular disease in patients with obstructive sleep apnea syndrome (OSAS), Folia Histochemica et Cytobiologica 49 (2011), 206-210.

[29] M. Tazbirek, L. Slowinska, S. Skoczynski and W. Pierzchala, Short-term continuous positive airway pressure therapy reverses the pathological influence of obstructive sleep apnea on blood rheology parameters, Clinical Hemorheology and Microcirculation 41 (2009), 241-249.

[30] D.N.Tziakas, G.K. Chalikias, I.K. Tentes, D. Stakos, S.V. Chatzikyriakou, K. Mitrousi, A.X. Kortsaris, J.C. Kaski and H. Boudoulas, Interleukin- 8 is increased in the membrane of circulating erythrocytes in patients with acute coronary syndrome, European Heart Journal 29 (2008), 2713-2722.

[31] N. Uyesaka, S. Hasegawa, N. Ishioka, R. Ishioka, H. Shio and A.N. Schechter, Effects of superoxide anions on red cell deformability and membrane proteins, Biorheology 29 (1992), 217-229.

[32] B. Wang, B. Yan, D. Son, X. Ye and S.F. Liu, Chronic intermittent hypoxia down-regulates endothelial nitric oxide synthase expression by an NF-kB-dependent mechanism, Sleep Medicine 14 (2013), 165-171.

[33] H.K. Yaggi, J. Concato, W.N. Kernan, J.H. Lichtman, L.M. Brass and V. Mohsenin, Obstructive sleep apnea as a risk factor for stroke and death, New England Journal of Medicine 353 (2005), 2034-2241.

[34] W. Zhang, Y. Sun, G. Zhang, Y. Wang and H. Sun, The effect of obstructive sleep apnea-hypopnea syndrome on acute myocardial infarction, Bratislaske Lekarske Listy 113 (2012), 565-568. 